\title{
Challenges in the use of tests to diagnose tuberculosis infection
}

\author{
The Editors \\ NSW Public Health Bulletin
}

\section{Dear Editors}

Congratulations on the publication of the "Tuberculosis in NSW" edition (2013; 24(1)). It makes excellent reading on a disease that continues to smoulder in this country. However, there is one topic on which some authors appear unclear: the limitations of immunological tests for both tuberculosis (TB) disease and infection. I refer to the tuberculin skin test (TST) and interferon-gamma release assays (IGRAs), in particular the QuantiFERON-Gold In Tube ${ }^{\circledR}$ (QFT-Gold IT). Certainly Britton et al are correct in stating the unreliability of such tests in infants under the age of 2. Indeed, these tests have little application in diagnosing disease, but are the only diagnostic agents we have for infection. It is not good enough for authors to tell us that the TST is negative or positive since this means nothing in an investigation where we have to balance sensitivity against specificity. A TST threshold of $10 \mathrm{~mm}$ induration might be said to achieve this balance, but the QFT-Gold IT shows that it does not. Across the world, tuberculins are produced that are of different potency, are recommended to be given in different doses (not always 10 units), and a "positive induration" may be less than $10 \mathrm{~mm}$. Therefore authors should be encouraged to tell us what dose of which tuberculin has achieved what degree of induration. Although IGRAs are reported as positive and negative, this is dependant on an arbitrary cut-off point. The criterion we use for a "positive" TST in Australia may be sensitive, but has poor specificity and for QFT-Gold IT, mediocre sensitivity, if good specificity. I am surprised that none of the articles dealing with TB infection mention the use of both tests being used together (except in the BCGvaccinated), a strategy that we in Australia can surely afford.

\section{John E. Thompson}

Editor, The Australian Tuberculosis Review

\section{The Editors}

NSW Public Health Bulletin

\section{Dear Editors}

Thompson agrees that both the tuberculin skin test (TST) and interferon-gamma release assays (IGRAs) have little application in diagnosing tuberculosis (TB) disease, although they provide important information about likely Mycobacterium tuberculosis infection, as does a careful TB exposure history. ${ }^{1,2}$ We acknowledge that choosing a TST induration cut-off represents a sensitivity and specificity trade-off. The use of variable cut-offs $(5,10$ or $15 \mathrm{~mm}$ ) depending on the likelihood of TB exposure (using lower values when the likelihood of M. tuberculosis infection is higher) has been advocated by the American Thoracic Society. ${ }^{3}$ However, this rationale does not apply to TB-endemic areas where TB exposure risk is near universal. The World Health Organization (WHO) promotes a single cut-off of $\geq 10 \mathrm{~mm}$ ( $\geq 5 \mathrm{~mm}$ in immunocompromised individuals), using 5 tuberculin units (TU) of purified protein derivative (PPD) or equivalent, such as 2TU RT-23 ${ }^{\circledR}$ (Statens Serum Institute, Denmark). ${ }^{4}$ New South Wales uses 5TU $\left(0.1 \mathrm{ml}\right.$ Tubersol ${ }^{\circledR}$; Sanofi Pasteur, Toronto, Canada) with WHO aligned cut-offs.

Since PPD includes multiple peptides that are also found in BCG vaccine and non-tuberculous mycobacteria, TST specificity is compromised. The QuantiFERON-Gold In Tube ${ }^{\circledR}$ test (QFT-Gold IT) has improved specificity, especially in BCG-vaccinated individuals, but not all discordant results can be ascribed to poor TST specificity. Suboptimal QFT-Gold IT sensitivity is a concern, particularly in young children, while indeterminate results and blood sampling (which requires $3 \mathrm{ml}$ of whole blood) pose additional challenges. ${ }^{5,6}$ In the United Kingdom, National Institute for Health and Clinical Excellence (NICE) guidelines previously recommended a two-step process using TST for screening and QFT-Gold IT for confirmation, but revised guidelines encourage clinicians to consider preventive therapy in high-risk individuals who test TST positive and QFT negative. ${ }^{7}$ Since the risk of disease progression, including disseminated forms of disease such as TB meningitis and miliary $\mathrm{TB}$, is highest in young children $(<2-$ 3 years of age), ${ }^{8}$ combined testing should be considered, with either positive test indicating likely $M$. tuberculosis infection. False-positive TST readings in BCG-vaccinated children remain problematic in this context. Research into better immunodiagnostic tests is a key research priority.

\section{Ben J. Marais}

Marie Bashir Institute for Infectious Disease and Biosecurity, University of Sydney and the Children's Hospital at Westmead, Sydney, Australia

\section{References}

1. Britton P, Perez-Velez CM, Marais BJ. Diagnosis, treatment and prevention of tuberculosis in children. NSW Public Health Bull 2013; 24(1): 15-21. doi:10.1071/NB12100 
2. National TB Advisory Committee. Position statement on interferon-gamma release assays in the detection of latent tuberculosis infection. Commun Dis Intell 2012; 36: 125-31.

3. American Thoracic Society. Targeted tuberculin testing and treatment of latent infection. Am J Respir Crit Care Med 2000; 161: S221-47. doi:10.1164/ajrccm.161.supplement_3.ats600

4. World Health Organization. Guidance for national tuberculosis programmes on the management of tuberculosis in children. Available at: http://whqlibdoc.who.int/hq/2006/ WHO_HTM_TB_2006.371_eng.pdf (Cited 1 October 2013).

5. Mandalakas AM, Detjen AK, Hesseling AC, Benedetti A, Menzies D. Interferon-gamma release assays and childhood tuberculosis: systematic review and meta-analysis. Int J Tuberc Lung Dis 2011; 15(8): 1018-32. doi:10.5588/ijtld.10.0631

6. Connell TG, Zar HJ, Nicol MP. Advances in the diagnosis of pulmonary tuberculosis in HIV-infected and HIV-uninfected children. J Infect Dis 2011; 204(Supp1 4): S1151-8. doi:10.1093/ infdis/jir413

7. National Institute for Health and Clinical Excellence (NICE) Tuberculosis: Clinical diagnosis and management of tuberculosis, and measures for its prevention and control (CG117). London: Royal College of Physicians; 2011. Available at: http://guidance.nice.org.uk/CG117 (Cited 1 October 2013).

8. Perez-Velez CM, Marais BJ. Tuberculosis in children. $N$ Engl $J$ Med 2012; 367: 348-61. doi:10.1056/NEJMra1008049

\section{The Editors}

\section{NSW Public Health Bulletin}

\section{Dear Editors}

I would like to thank Dr Thompson for his comments regarding limitations of diagnostic tests for latent tuberculosis infection (LTBI). It is true that a universal cut-off level of $10 \mathrm{~mm}$ induration to define a positive or negative tuberculin skin test (TST) (and thus the presence or absence of LTBI), as used in the NSW tuberculosis (TB) contact study, ${ }^{1}$ does not account for individual variations. To establish whether infection is truly present the pre-test probability of a person having LTBI has to be taken into account together with the result of a screening test for LTBI: TST or interferon-gamma release assays (IGRAs). The pre-test probability is based on the exposure to TB depending on the incidence of TB in the country of residence (and in the country of origin, if different) as well as on the TB contact status (no contact, casual or close contact). In interpreting the actual test, the size of the TST reaction and whether a TST conversion occurred will influence the likelihood that true LTBI is present. Potential reasons for a false-positive TST or a false TST conversion, such as recent or multiple BCG vaccination(s), exposure to non-tuberculous mycobacteria and immunological boosting due to a previous TST in a BCG-vaccinated individual, have to be considered. Likewise, potential reasons for a false-negative TST or IGRA, such as conditions associated with anergy and a short time interval since exposure occurred, have to be taken into account when trying to establish whether true LTBI is present. While all those factors warrant consideration in clinical practice, such a highly individualised approach of determining whether true LTBI is present is not feasible when presenting data from a retrospective cohort study with more than 14000 TB contacts. A TST result of $10 \mathrm{~mm}$ has a sensitivity of $90 \%$ and specificity of $>95 \%$, and is the recommended cut-off point for most clinical situations. ${ }^{2}$ It is thus reasonable to use this cut-off to define LTBI in a study setting. In the NSW TB contact study all TB contacts were tested with the same tuberculin (5 tuberculin units, $0.1 \mathrm{ml}$ Tubersol ${ }^{\circledR}$; Sanofi Pasteur).

IGRAs were not routinely used in the examined cohort of TB contacts, but I agree with Thompson that a combination of TST and IGRA can be used not only to confirm a positive TST in a BCG-vaccinated low-risk person, but also to enhance the overall sensitivity in people with a high risk of infection and/or progression to disease. In this context, the tests could be used sequentially (in any order) after an initial test was negative or indeterminate/borderline.

IGRAs are not recommended if repeat testing is planned, for example in health care workers with potentially ongoing TB exposure, as studies on serial IGRA testing have shown high rates of conversions and reversion, independent of exposure or treatment. ${ }^{3,4}$ Routine dual testing with both TST and IGRA is thus not indicated.

\section{Claudia C. Dobler}

Woolcock Institute of Medical Research, University of Sydney and Liverpool Hospital, Sydney

\section{References}

1. Dobler CC. What do we know about the outcomes of tuberculosis contact investigations in NSW? N S W Public Health Bull 2013; 24(1): 34-7. doi:10.1071/NB12099

2. Menzies RI. Tuberculin skin testing. In: Reichman LB, Hershfield ES, eds. Tuberculosis: A Comprehensive International Approach. New York: Marcel Dekker; 2000. p. 279-322.

3. Metcalfe JZ, Cattamanchi A, McCulloch CE, Lew JD, Ha NP, Graviss EA. Test variability of the QuantiFERON-TB gold in-tube assay in clinical practice. Am J Respir Crit Care Med 2013; 187(2): 206-11. doi:10.1164/rccm.201203-0430OC

4. Slater ML, Welland G, Pai M, Parsonnet J, Banaei N. Challenges with QuantiFERON-TB Gold Assay for Large-Scale, Routine Screening of US Healthcare Workers. Am J Respir Crit Care Med 2013; 188(8): 1005-10. doi:10.1164/rccm.201305$08310 \mathrm{C}$ 